\title{
The Evolution of Continuous Variation in Ejaculate Expenditure Strategy
}

\author{
Samuel J. Tazzyman, ${ }^{1,2, *}$ Tommaso Pizzari, ${ }^{3}$ Robert M. Seymour, ${ }^{1,4}$ and Andrew Pomiankowski ${ }^{1,2}$ \\ 1. CoMPLEX (Centre for Mathematics and Physics in the Life Sciences and Experimental Biology), University College London, Gower \\ Street, London WC1E 6BT, United Kingdom; 2. Galton Laboratory, Research Department of Genetics, Environment and Evolution, \\ University College London, 4 Stephenson Way, London NW1 2HE, United Kingdom; 3. Edward Grey Institute, Department of \\ Zoology, University of Oxford, South Parks Road, Oxford OX1 3PS, United Kingdom; 4. Department of Mathematics, University \\ College London, Gower Street, London WC1E 6BT, United Kingdom
}

Submitted November 13, 2008; Accepted March 19, 2009; Electronically published July 23, 2009

\begin{abstract}
Sperm competition theory has largely focused on the evolution of ejaculate expenditure strategies across different species or populations or across discrete mating roles on which sperm competition operates differentially. Few studies have considered the extent to which male ejaculate expenditure is influenced by continuous change in male phenotype within a population. Here we model how optimal ejaculate expenditure responds to two sources of continuous variation: (1) the quantity of resources allocated by a male to mating within a breeding season and (2) the resource cost of obtaining a mate. We find that variation in the amount of resources available for mating does not alone produce selection for differing ejaculate investment strategies. However, when there is variation in the cost of obtaining a mate, males with a lower cost will be selected to invest fewer sperm per mating than males whose cost is higher. Any parameter decreasing this cost will also select for decreased ejaculate investment per mating. These results provide a novel insight into the evolution of male ejaculate expenditure strategies, revealing that individual constraints on the ability to secure matings can lead to variation in ejaculate expenditure even when the risk of sperm competition is the same for all males.
\end{abstract}

Keywords: sperm competition, sexual selection, ornaments, fertility.

\section{Introduction}

In many species, females mate multiply and the ejaculates of different males compete to fertilize a set of eggs, a process called sperm competition (Parker 1970; Pizzari and Parker 2009). A key determinant of the outcome of sperm competition is the relative number of sperm delivered by competing ejaculates (Martin et al. 1974; Wedell et al. 2002; Gage and Morrow 2003), generating postinsemination sexual selection on male investment in the number of sperm inseminated (ejaculate expenditure). Sperm com-

* Corresponding author; e-mail: s.tazzyman@ucl.ac.uk.

Am. Nat. 2009. Vol. 174, pp. E71-E82. (C) 2009 by The University of Chicago. 0003-0147/2009/17403-50870\$15.00. All rights reserved.

DOI: $10.1086 / 603612$ petition theory has traditionally modeled strategies of ejaculate expenditure in a species or population as the response to the average degree of sperm competition faced by an ejaculate (Parker 1990a, 1998; Parker et al. 1996; Wedell et al. 2002). This view has been challenged by recent studies (Parker and Ball 2005; Williams et al. 2005) that propose a more dynamic alternative in which optimal ejaculate expenditure and the degree of sperm competition coevolve within a population, each having an effect on the other. This interdependence arises because as the optimal ejaculate expenditure increases, the expected number of matings per male decreases. Therefore, the expected number of matings per female will also decrease and with it the degree of sperm competition that an ejaculate is expected to face. It is this feedback that determines the evolutionary outcome.

Models of sperm competition have generally assumed that differences between males within a species or population are negligible compared to those between species or populations. Where variation within species or populations has been investigated, the male population has been split into discrete reproductive strategies that bias the level of sperm competition faced by the ejaculates of different males, bias the information males have on the level of sperm competition associated with a copulation, or "load" the sperm competition raffle between the ejaculates of males mating with the same female in different roles (Parker 1990a, 1990b; Mesterton-Gibbons 1999a, 1999b; Ball and Parker 2000). These studies predict that each male reproductive strategy, or role, will have a corresponding discrete optimal ejaculate expenditure strategy. However, much phenotypic variation among males is likely to be continuous because of, for example, variable access to resources, female mate choice, environmental or genetic factors, and differences in age or phenotype. Despite its biological generality, the potential effect of continuous, within-population variation on ejaculate expenditure has 
not been explicitly modeled. In this article, we provide a starting point for an analysis of such effects.

We adapt the alternative theoretical model for ejaculate expenditure (Williams et al. 2005) to include within-population variation, and we consider two candidate continuously varying traits. First, the quantity of resources allocated to reproduction $(R)$ may vary continuously across a population of males. Some males may be able to obtain more resources overall and thus have proportionately more for reproduction. Alternatively, males may allocate resources differently in each breeding season throughout their lifetime (e.g., yearlings vs. mature adults; Pianka and Parker 1975). In either case, the quantity of resources available for reproductive effort in a given breeding season will differ from male to male, and we expect this to vary in a continuous manner.

Second, males may differ in the resource cost $(c)$, that is, the time and energy they spend seeking out and courting a female in order to obtain a mating. One mechanism that could result in such variation is female mate choice. For example, if females display preference toward certain male phenotypic traits, a male will pay progressively less to mate as his phenotype approaches that preferred by females (Andersson 1994; Andersson and Iwasa 1996). Similarly, males may vary continuously in their competitive ability to gain access to females. In this case, a male with a higher $c$ value must work harder to secure a mating than one with a lower $c$ value. Again, we expect that this cost will vary continuously across a population. Note that the amount of resources a male puts into the growth and maintenance of a secondary sexual ornament or a position in a social hierarchy in order to attract females is a cost distinct from that being modeled here. For the purposes of this article, the cost a male pays to attract a female is simply a measure of the mean amount of effort he has to expend to attract each mate in a given breeding season. This may be related to a male's investment in attractiveness or competitive ability, but the cost of this initial investment is assumed to have been paid before the breeding season begins. Thus, all males arrive in our model at the start of the breeding season with their $c$ and $R$ values already in place. We do, however, allow for the possibility that these parameters are not independent.

\section{The Model}

Let $R$ be the quantity of resources allocated by a male to reproduction in a given mating season. Suppose that it costs a male $c$ units of resource to obtain a mate and that for every mating a further $s$ units of resource are used for the production of sperm. For simplicity, we assume that a male's ejaculate expenditure is the same for every mating in a breeding season (the validity of this assumption is considered in "Discussion"). The total cost per mating is therefore $c+s$. A male playing a strategy $s$ then has an expected number of matings $n(s)$, where

$$
n(s)=\frac{R}{c+s} .
$$

In order to calculate the relative fitness $W(s, \hat{s})$ of a mutant strategy $s$ against a population of males all playing strategy $\hat{s}$, we calculate its expected reproductive success $\nu(s, \hat{s})$ per mating. Then the expected reproductive success is

$$
W(s, \hat{s})=n(s) \nu(s, \hat{s})
$$

the product of the expected number of matings and the expected success per mating (Parker 1998; see also Mesterton-Gibbons 1999a, $1999 b$ and Ball and Parker 2000 for discussions of alternative additive models).

To calculate $\nu(s, \hat{s})$, we assume that sperm competition is a fair raffle (Parker 1982, 1990a, 1990b, 1998) and that the "fertilizing power" of the sperm produced by a male is linearly proportional to the resource expenditure $s$ that the male invests in producing the sperm. Thus, putting twice as many resources into an ejaculate is assumed to double its competitive efficiency, perhaps because there are twice as many sperm in the ejaculate (we consider the generality of this assumption in "Discussion"). The expected reproductive success of a mutant male playing strategy $s$ from mating with a female who also mates with $k$ wild-type males playing strategy $\hat{s}$ will simply be the proportion of his sperm inseminated in the female, $s /(s+$ $k \hat{s})$.

We now need to know the probability $p_{k}$ that a female who mates with a focal male will have mated with $k$ additional males in the breeding season. In a large population with unitary sex ratio, random mating, and a negligible chance that any male will mate with the same female twice, this probability is given by the Poisson distribution

$$
p_{k}=\frac{\hat{n}^{k} e^{-\hat{n}}}{k !}
$$

where $\hat{n}$ is the expected number of matings achieved by a male playing strategy $\hat{s}^{1}$ Therefore, the expected reproductive success $\nu(s, \hat{s})$ of a male playing strategy $s$ against a population playing strategy $\hat{s}$ is

\footnotetext{
${ }^{1}$ Note that Williams et al. (2005) use the probability $q_{k}$ that a male mates with a female who mates with $k-1$ other males, for $k=1,2,3, \ldots$. Thus, their $q_{k}$ is the same as our $p_{k-1}$.
} 


$$
\nu(s, \hat{s})=\sum_{k=0}^{\infty} p_{k} \frac{s}{s+k \hat{s}} .
$$

We now adopt an evolutionarily stable strategy (ESS) approach (Maynard Smith 1974, 1982). We look for an ESS $s^{*}$ that is a strict best reply to itself. In other words, against a population of males playing strategy $s^{*}$, any mutant male playing an alternative strategy $s \neq s^{*}$ will have strictly lower fitness than the wild type playing $s^{*}$. Mathematically, this states that $W\left(s^{*}, s^{*}\right)>W\left(s, s^{*}\right)$ for any $s \neq s^{*}$. Note that, usually, an ESS $s^{*}$ requires the weaker condition that $W\left(s^{*}, s^{*}\right) \geq W\left(s, s^{*}\right)$ for any $s \neq s^{*}$ and that for any mutant strategy $s$ such that $W\left(s^{*}, s^{*}\right)=W\left(s, s^{*}\right)$ we also have $W\left(s^{*}, s\right)>W(s, s)$. However, here there will be no alternative best replies, since we show in appendix A that $\left.\left(\partial^{2} W / \partial s^{2}\right)\right|_{s=s^{*}}<0$, and so the ESS is a nondegenerate maximum (cf. Williams et al. 2005).

\section{Variation in Resources and Costs}

We assume a fixed distribution of resource allocation $R$ and cost $c$ over the population of males. A male with resources $R$ and cost $c$ (referred to as an $(R, c)$ male) follows ejaculate investment strategy $s(R, c)$ and has a corresponding expected number of matings $n(R, c)$ :

$$
n(R, c)=\frac{R}{c+s(R, c)} .
$$

We denote the population mean values for ejaculate investment and expected number of matings by $\bar{s}$ and $\bar{n}$, respectively. If an $(R, c)$ male plays strategy $s$ against a population of males with mean strategy $\bar{s}$ and mean number of matings $\bar{n}$, his fitness $W(R, c, s \mid \bar{s}, \bar{n})$ is then calculated by substituting equations (2)-(4) into equation (1), giving

$$
W(R, c, s \mid \bar{s}, \bar{n})=\frac{R}{c+s} \sum_{k=0}^{\infty} \frac{\bar{n}^{k} e^{-\bar{n}}}{k !} \frac{s}{s+k \bar{s}} .
$$

We wish to find the ESS ejaculate expenditure $s^{*}$ for the population as a function of $R$ and $c$. This is the strategy $s^{*}(R, c)$, such that for any pair $R$ and $c$ and any $s \neq$ $s^{*}(R, c)$,

$$
W\left(R, c, s \mid \bar{s}^{*}, \bar{n}^{*}\right)<W\left(R, c, s^{*}(R, c) \mid \bar{s}^{*}, \bar{n}^{*}\right)
$$

so an $(R, c)$ male in a heterogeneous male population whose ejaculate expenditure is determined by the function $s^{*}$ can lower his fitness only by using a strategy other than $s^{*}(R, c)$ (see app. A for proof that the ESS is a nondegen- erate maximum and thus that there can be no alternative best reply).

\section{Risk and Intensity of Competition}

In some previous theoretical work (Parker 1998), the ejaculate expenditure strategy of a male has been expected to vary with the risk or intensity of sperm competition. Here we follow Williams et al. (2005) and define the risk of competition for a focal ejaculate as the probability that sperm competition occurs $\left(1-e^{-\bar{n}^{*}}\right.$ at the ESS, since $e^{-\bar{n}^{*}}$ is the probability that a female receives no other matings) and the intensity of competition as the number of ejaculates expected to compete for paternity of a given female's offspring ( $\bar{n}^{*}$ at the ESS). By these definitions, all males face the same risk and intensity of sperm competition. Any differences in optimal ejaculate expenditure strategy will therefore not be due to the risk or intensity differences outlined in classical models.

\section{Results \\ Analysis}

An ESS for an $(R, c)$ male with quantity of resources $R$ and cost of obtaining a mate $c$ will be a solution of the equation

$$
\frac{\partial W}{\partial s}\left(R, c, s \mid \bar{s}^{*}, \bar{n}^{*}\right)=0 .
$$

Assuming that such an ESS exists, ${ }^{2}$ we differentiate equation (5) with respect to $s$ at $\bar{s}=\bar{s}^{*}$ and $\bar{n}=\bar{n}^{*}$, to obtain

$$
\begin{aligned}
\frac{\partial W}{\partial s}\left(R, c, s \mid \bar{s}^{*}, \bar{n}^{*}\right)= & \frac{R c}{(c+s)^{2}} \sum_{k=0}^{\infty} \frac{\left(\bar{n}^{*}\right)^{k} e^{-\bar{n}^{*}}}{k !} \frac{1}{s+k \bar{s}^{*}} \\
& -\frac{R s}{(c+s)} \sum_{k=0}^{\infty} \frac{\left(\bar{n}^{*}\right)^{k} e^{-\bar{n}^{*}}}{k !} \frac{1}{\left(s+k \bar{s}^{*}\right)^{2}} \\
= & 0,
\end{aligned}
$$

which implies that

\footnotetext{
${ }^{2}$ It can be shown analytically that such an ESS exists under weak assumptions on the joint distribution of $R$ and $c$ over the male population. The analysis can be extended more generally to cover distributions over any set of male characteristics that influence cost, specified by parameters $g_{1}, \ldots, g_{i}, \ldots$, with $g_{1}=R$ and $c=c\left(g_{1}, \ldots, g_{i}, \ldots\right)$, a function of these parameters. However, the proof is outside the scope of this article. Instead, we construct illustrative examples of the ESS by using an iterative numerical procedure; see figures 2, 3.
} 


$$
\begin{aligned}
\frac{c}{\left(c+s^{*}\right)} \sum_{k=0}^{\infty} \frac{\left(\bar{n}^{*}\right)^{k} e^{-\bar{n}^{*}}}{k !} \frac{1}{s^{*}+k \bar{s}^{*}} & \\
& =s^{*} \sum_{k=0}^{\infty} \frac{\left(\bar{n}^{*}\right)^{k} e^{-\bar{n}^{*}}}{k !} \frac{1}{\left(s^{*}+k \bar{s}^{*}\right)^{2}} .
\end{aligned}
$$

This final rearrangement no longer includes $R$, and so the ESS ejaculate investment strategy will not depend on $R$ unless the cost $c$ depends on $R$.

Equation (6) can be solved to obtain $c$ as a function of $s, \bar{s}^{*}$, and $\bar{n}^{*}$ :

$$
c\left(s \mid \bar{s}^{*}, \bar{n}^{*}\right)=\frac{\sum_{k=0}^{\infty}\left[\left(\bar{n}^{*}\right)^{k} e^{-\bar{n}^{*}} / k !\right]\left[s /\left(s+k \bar{s}^{*}\right)\right]^{2}}{\sum_{k=0}^{\infty}\left[\left(\bar{n}^{*}\right)^{k} e^{-\bar{n}^{*}} / k !\right]\left[k \bar{s}^{*} /\left(s+k \bar{s}^{*}\right)^{2}\right]} .
$$

Now, $c\left(s \mid \bar{s}^{*}, \bar{n}^{*}\right)>0$ for all $s \geq 0$, and $c(s)$ is monotonically increasing with $s$ (because each summand in the numerator is monotonically increasing and each summand in the denominator is monotonically decreasing). Therefore, there is a unique inverse function $s\left(c \mid \bar{s}^{*}, \bar{n}^{*}\right)$, defined for $c \geq 0$, that is also monotonically increasing. This function is the ESS $s^{*}$.

Thus, the ESS ejaculate investment strategy $s^{*}$ is monotonically increasing in $c$ and depends on $R$ only insofar as $c$ depends on $R$ (e.g., if $c$ is a decreasing function of $R$, then $s^{*}$ will also be a decreasing function of $R$ ). Biologically, this means that a male's optimal ejaculate expenditure will not vary with respect to the quantity of resources allocated to reproduction unless the cost that males pay to obtain a mating also varies with respect to quantity of resources. On the other hand, as a male's expected cost of obtaining a mating increases, his optimal ejaculate expenditure per mating also increases. This makes sense intuitively: males that have to pay a higher cost in resource expenditure in order to obtain a mating (high- $c$ males) will value that mating more highly and thus will invest more sperm. Such males expect to obtain fewer matings, and therefore in this way they exploit the small number of opportunities they get to maximize their overall reproductive success. In contrast, males that have to pay a relatively low cost to obtain a mating (low- $c$ males) will not value each mating so highly and will benefit by investing fewer sperm per mating. By doing this they exploit the large number of matings they gain, more than making up for the lower expected share of paternity per mating.

This can be illustrated using the marginal-value theorem (Charnov 1976). Males want to maximize their overall fitness, which is $\nu(s) \times R /(c+s)$. Since $\nu(s)$ is a sum of increasing concave functions, it is itself increasing and concave (see app. A for further details). The optimal ejaculate expenditure strategy will increase with $c$ but will be independent of $R$. In summary, the harder it is for a male to obtain a mating, the more sperm he will invest in that mating (fig. 1).

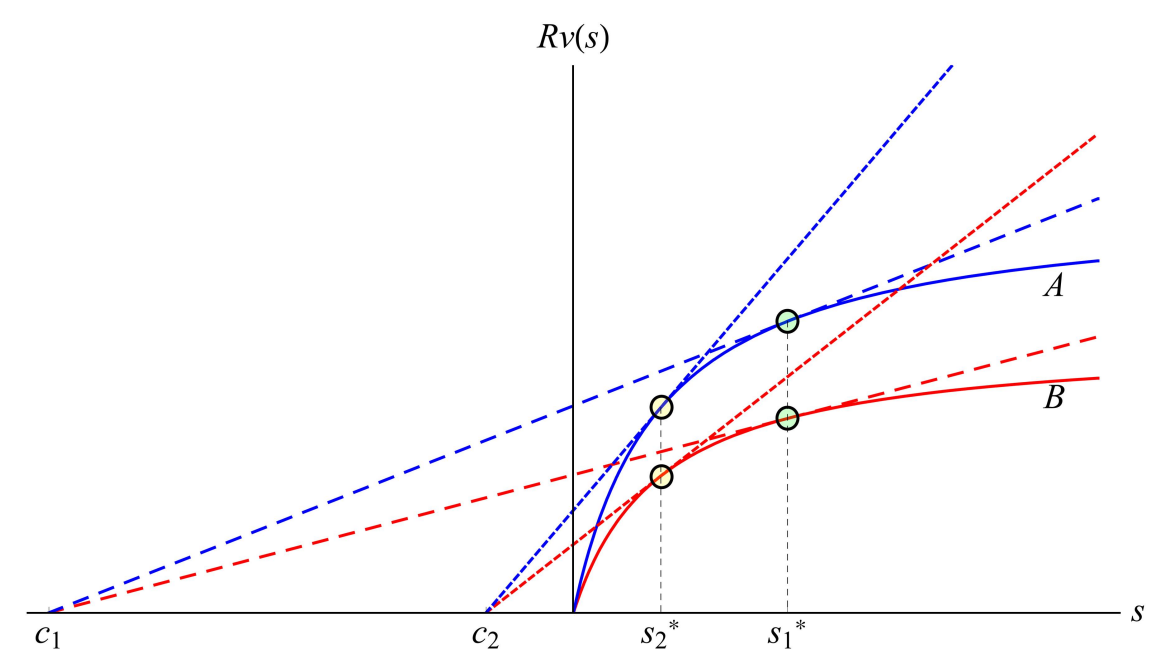

Figure 1: Illustration that the optimal ejaculate investment strategy $s^{*}$ increases with resource cost $(c)$ but is independent of reproduction $(R)$. The solid lines represent the expected reproductive success per mating multiplied by the quantity of resources for two males, $A$ and $B$. Both males are competing in the same population and thus against the same population mean ejaculate investment strategy $\bar{s}$ and population mean number of matings $\bar{n}$. Male $A$ has more resources than male $B$. Both males attempt to optimize their fitness, which is equal to $R \nu /(c+s)$, which amounts to maximizing the gradients of the dashed lines. If the two males have the same cost (i.e., both have $\operatorname{cost} c_{1}$ or $c_{2}$ ), then their optimal ejaculate investment strategy is the same $\left(s_{1}^{*}\right.$ or $s_{2}^{*}$ ), despite having differing amounts of resources. If they have differing costs (one has cost $c_{1}$ and the other cost $c_{2}$ ), then they will have differing optimal ejaculate investment strategies $\left(s_{1}^{*}\right.$ or $s_{2}^{*}$, corresponding to $\operatorname{cost} c_{1}$ and $c_{2}$, respectively). 


\section{Numerical Simulations}

We used an iterative method that converges on the optimal strategy (see app. B for details) to numerically calculate some explicit examples of evolutionarily stable ejaculate investment strategies. For clarity of presentation, we broke the problem down into two discrete cases, one in which all males have the same $c$ value but vary in $R$ and one in which all males have the same $R$ value but vary in $c$.

Variation in $\mathrm{R}$. For a fixed value of $c$ common to all males, we considered a distribution of $R$ values across the male population specified by a truncated normal distribution. Three example ESSs are shown in figure 2. As expected, for all three sets of parameters the evolutionarily stable ejaculate expenditure strategy $s^{*}$ does not vary with $R$. Since $n(R)=R /\left(c+s^{*}\right)$, it follows that the expected number of matings for a male increases linearly with the quantity of resources he allocates to mating. Thus, males with more resources obtain more matings because they are able to "stay the course" longer.

With all males following the same ejaculate expenditure strategy $s=\bar{s}^{*}$, the fitness function (eq. [5]) becomes

$$
W\left(R, c, \bar{s}^{*} \mid \bar{s}^{*}, \bar{n}^{*}\right)=\frac{R\left(1-e^{-\bar{n}^{*}}\right)}{\bar{n}^{*}\left(c+\bar{s}^{*}\right)},
$$

and a male's fitness is proportional to the resources he allocates to breeding. Therefore, lower- $R$ males can never achieve a fitness as high as that of higher- $R$ males.

Using this method, we also investigated how changing the population mean quantity of resources allocated to reproduction $\bar{R}$ affects the solution to the model when the minimum and maximum $R$ values are fixed (fig. 2). We found that the optimal ejaculate expenditure for all males increases (decreases) with increasing (decreasing) $\bar{R}$. Thus, although a male's individual quantity of resources does not affect his optimal ejaculate expenditure, the population mean quantity of resources does. To see mathematically why this occurs, see appendix C. Biologically, this makes sense because if males, on average, have more resources for mating and the cost of mating remains the same, then they profit by investing the extra resources in ejaculate expenditure.

Variation in c. For a fixed value of $R$ common to all males, we considered a distribution of $c$ values across the male population specified by a truncated normal distribution. Three example ESSs are shown in figure 3. As expected, for all three sets of parameters, the ESS $s^{*}(c)$ is an increasing function of $c$.

We also note that if $R$ is fixed for all males, then a higher- $c$ male can never have a fitness higher than that of a lower- $c$ male. To see this, consider a population playing ESS mean ejaculate expenditure strategy $\bar{s}^{*}$, with a corresponding mean number of matings $\bar{n}^{*}$. Suppose we have

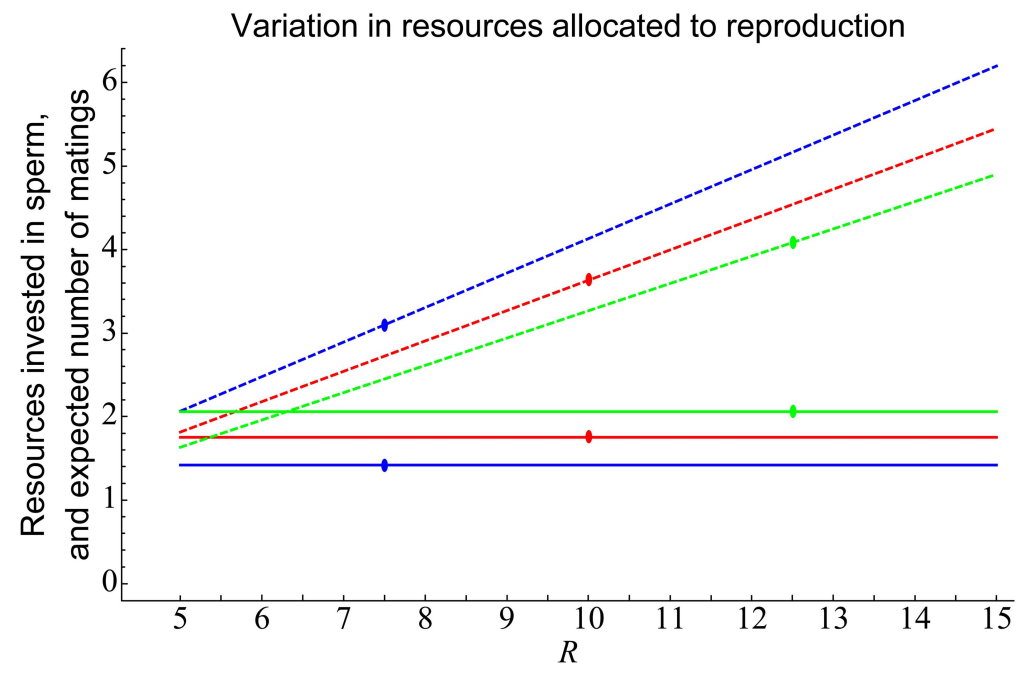

Figure 2: Evolutionarily stable optimal ejaculate expenditure strategies $s^{*}(R)$ (solid lines) and corresponding expected numbers of matings $n^{*}(R)$ (dashed lines) plotted against total quantity of resources allocated to reproduction $(R)$. The position of the mean male is marked on each curve. For each curve, $R$ values are normally distributed (truncated between 5 and 15) across the population, with SD $(\sigma)$ of 1.5 and means of 7.5 (blue), 10 (red), and 12.5 (green). The cost of obtaining a mate is $c=1$ for all males. The functions were obtained with the numerical iterative method described in appendix B. 


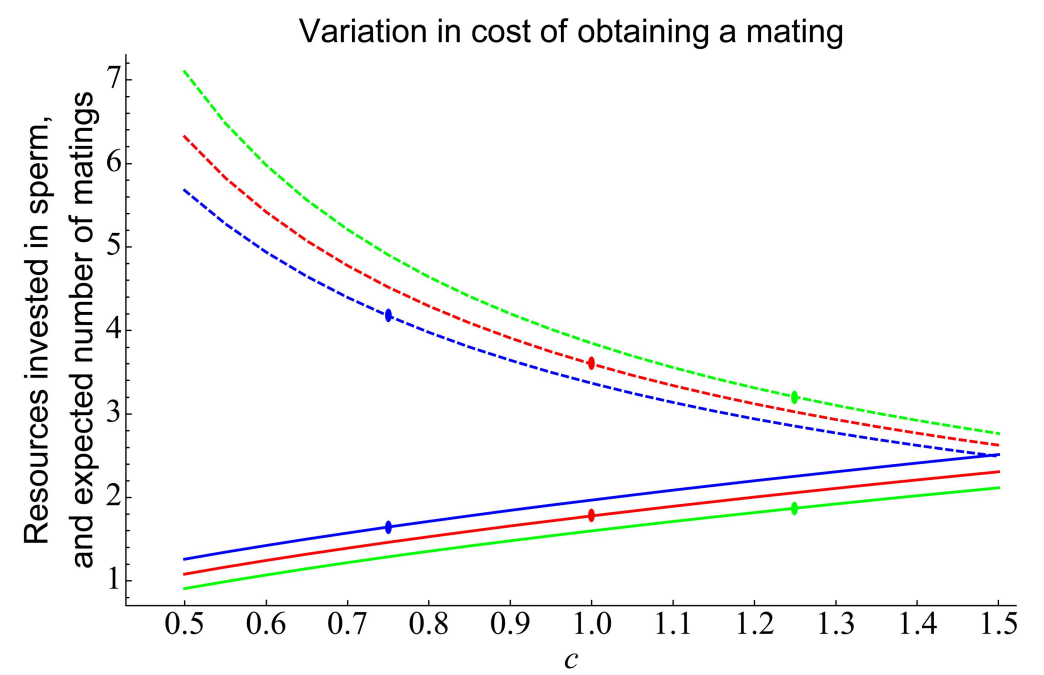

Figure 3: Evolutionarily stable optimal ejaculate expenditure strategies $s^{*}(c)$ (solid lines) and corresponding expected numbers of matings $n^{*}(c)$ (dashed lines) plotted against cost of obtaining a mating $(c)$. The position of the mean male is marked on each curve. For each curve, $c$ values are normally distributed across the population (truncated between 0.5 and 1.5), with SD $(\sigma)$ of $1 / 6$ and means of 0.75 (blue), 1 (red), and 1.25 (green). The quantity of resources allocated to reproduction is $R=10$ for all males. The functions were obtained with the numerical iterative procedure described in appendix B.

two males, with costs of obtaining a mating $c_{1}$ and $c_{2}$, where $c_{1}<c_{2}$. By definition, both males will achieve their maximum fitness only by playing the ESS strategies $s^{*}\left(c_{1}\right)$ and $s^{*}\left(c_{2}\right)$. Thus, since expression (5) decreases as $c$ increases for fixed $s$, it follows that

$$
\begin{aligned}
W\left(R, c_{2}, s^{*}\left(c_{2}\right) \mid \bar{s}^{*}, \bar{n}^{*}\right) & <W\left(R, c_{1}, s^{*}\left(c_{2}\right) \mid \bar{s}^{*}, \bar{n}^{*}\right) \\
& <W\left(R, c_{1}, s^{*}\left(c_{1}\right) \mid \bar{s}^{*}, \bar{n}^{*}\right)
\end{aligned}
$$

and so fitness decreases as cost $c$ increases.

We also investigated the effects of changing the population's mean cost of obtaining a mate when the minimum and maximum $c$ values were fixed (fig. 3). As illustrated, the ejaculate expenditure strategy for a focal male decreases as the population mean cost $\bar{c}$ increases. Thus, when males are in a population in which, on average, they find it harder to obtain a mate (represented by a higher mean cost $\bar{c}$ ), the optimal ejaculate expenditure strategy drops. To see why, imagine a focal male with some fixed cost of mating $c_{0}$, and consider the status of this male in two different populations with mean cost values $\bar{c}_{1}$ and $\bar{c}_{2}$, where $\bar{c}_{1}>$ $\bar{c}_{2}$. Our focal male, regardless of the value of $c_{0}$, will be relatively more attractive in the $\bar{c}_{1}$ population, where the average cost per mating is higher, than in the $\bar{c}_{2}$ population, where the average cost per mating is lower, and therefore will invest fewer resources in ejaculate expenditure in the former case than the latter. Since this is true for all values of $c_{0}$, it will be true for all males, and thus it explains why we see the optimal ejaculate expenditure strategy decrease as the mean cost value $\bar{c}$ rises.

\section{Discussion}

This study investigates how ejaculate expenditure strategies evolve across a continuously varying phenotypic gradient within a single population of males, under the assumptions that sperm competition occurs as a fair raffle with no perceptible differences among females (e.g., degree of polyandry, fecundity). The first general conclusion we draw is that differences in ejaculate expenditure strategies can evolve even within populations of males playing the same reproductive strategy. Sperm competition theory has focused on ejaculate expenditure strategies in relation to sperm competition risk or intensity (Parker 1998). While these are obviously important in shaping ejaculate expenditure across populations, our model shows that within a population there can be variation in optimal ejaculate expenditure even when all males face the same risk and intensity of competition in every mating.

To investigate this new theoretical framework, we considered two potential sources of continuous variation: the cost of obtaining a mate $(c)$ and the total resources allocated to reproduction $(R)$. Our second general conclusion is that variation in the cost of obtaining a mating alone is sufficient to cause differences in optimal ejaculate expenditure strategy, whereas variation in resources allocated to reproduction is not. This can be illustrated by the mar- 
ginal-value theorem (fig. 1; Charnov 1976). When a male obtains matings more easily than his rivals, he will value each mating less and thus will invest fewer sperm per mating. However, when males all pay the same cost to achieve matings, they all have the same evolutionarily stable ejaculate expenditure strategy, irrespective of the resources at their disposal.

We also investigated the effects of changing the population means of the two candidate traits (i.e., $R$ and $c$ ) on the optimal ejaculate expenditure strategy (figs. 2, 3). We found that, in the case of varying mating resources $R$, the optimal ejaculate expenditure increases with the amount of resources that the population, on average, allocates to reproduction. This is because if, on average, males have more resources available for reproduction and pay the same cost to obtain a mating, they invest the extra resources in ejaculate expenditure (Gage and Cook 1994). By contrast, in the case of varying mating cost $c$, optimal ejaculate expenditure for a given male decreases as the population mean cost of obtaining a mate increases. This is because a given male with a fixed cost of obtaining a mating will be relatively more attractive in a population with a higher mean cost than in one with a lower mean cost.

Our numerical modeling shows that less attractive males (higher $c$ ) cannot achieve a fitness as high as that of more attractive males (lower $c$ ) by changing their ejaculate expenditure strategy alone, if they have the same quantity of resources. Similarly, in the varying-resources $(R)$ model, at the ESS males with higher $R$ values have more resources to spend on mating overall and thus end up with higher fitness, because a male's expected fitness is proportional to his $R$ value (if all males have the same cost $c$ ). Thus, in both cases, the least successful males cannot catch up with their more successful rivals through differential ejaculate expenditure alone. If they are to compete successfully, these males will need to adopt some other tactics. In this way, selection could favor the evolution of alternative reproductive strategies (Shuster and Wade 2003) or other adaptations used by males to defend their paternity, for example, nonfertilizing sperm (Cook and Wedell 1999; Pizzari and Foster 2008), nonsperm ejaculate compounds such as seminal fluid peptides (Ram and Wolfner 2007), nonspermic copulations (Løvlie et al. 2005), mate guarding (Alcock 1994), the imposition of fitness costs associated with mating (Clutton-Brock and Parker 1995; Johnstone and Keller 2000; Lessells 2005), and, possibly, mating plugs (Simmons 2001; Moreira et al. 2007).

The value of the cost of obtaining a mating could arise from female preference, with low- $c$ males being those with whom females prefer to mate. However, this is likely to engender a dynamic scenario because low- $c$ males may impose fitness costs on their partners. First, our model suggests that males more likely to be chosen invest less per ejaculate. Thus, the most attractive males may not be able to fertilize all the eggs of the females that they are able to attract (e.g., Warner et al. 1995), generating intersexual conflict over remating rates (Alonzo and Warner 1999; Pizzari and Snook 2004). Second, mating with low$c$ males may also be associated with a higher risk of contracting sexually transmitted diseases (Graves and Duvall 1995). Together, these mating costs generate selection for females to avoid-rather than mate with-attractive males. When the intensity of such selection exceeds the direct or indirect benefits promoting female mating with these males, female preference might cease to favor attractive males. Therefore, continuously varying ejaculate expenditure strategies within a population could have important consequences for the evolution of female preference.

In turn, female behavior may drive the evolution of ejaculate expenditure strategies. The model investigated here assumes that there is no relationship between a male's $c$ value and the promiscuity of his mate, since all males mate randomly with females whose promiscuity is distributed according to the Poisson distribution. In reality, there may be some feedback in this system. Females mated to attractive, low- $c$ males might have a lower propensity to mate again, compared to females mated to unattractive, high- $c$ males. An example of such a mechanism in socially monogamous birds is when females paired with a poorquality social partner "trade up" through extra-pair copulations with males of more attractive phenotypes or more compatible genotypes (e.g., Jennions and Petrie 2000). The effect of this on the model would be for the optimal ejaculate expenditure of low- $c$ males to decrease yet further, as they would be less likely to face sperm competition. Conversely, the optimal ejaculate expenditure of high- $c$ males would increase, as they would be more likely to face greater sperm competition. This change would be in line with classical ideas about sperm competition risk (Wedell et al. 2002; Gage and Morrow 2003). However, the relationship between female promiscuity and $c$ value may go in the opposite direction, for example, if females mate more often to reduce infertility costs associated with attractive males. In this case, females might be directly selected to remate, and the increased risk of sperm competition faced by low- $c$ males would counterbalance the reduced costs of obtaining mates. It would be interesting to evaluate the relative strength of these selective forces. Clearly, the evolution of ejaculate expenditure strategies must be considered within a dynamic context in which competing male mating strategies coevolve with each other and with female responses (Moore and Pizzari 2005; Parker and Ball 2005; Gavrilets and Hayashi 2006).

In addition, for simplicity our model assumes that a 
male's ejaculate expenditure is the same for every mating in a given breeding season. It is important to note that this assumption has biological relevance and is consistent with classic sperm competition theory. Constant ejaculate expenditure tailored to the average level of sperm competition in the population is expected to arise whenever information on the level of sperm competition associated with individual mating opportunities is unavailable to males (Parker 1998). Even under loaded-raffle dynamics, the ejaculate expenditure of a male is not expected to vary across matings if males have no information on individual mating roles and have similar probabilities of mating in different roles at each mating (Parker 1998). However, it is possible that differential rates of sperm depletion may, in principle, affect ejaculate expenditure, which would expose low- $c$ males to high remating rates and a higher risk of sperm depletion (see above).

Finally, our numerical modeling considers the cost of obtaining a mate and the quantity of resources allocated to mating as distinct, uncorrelated categories of continuous variation. In the real world, these two parameters could be linked. For example, males who invest heavily in secondary sexual ornaments and thus pay a low cost to obtain a mate may have fewer resources available for breeding. Under these circumstances, we would expect low $c$ values to correspond to low $R$ values. On the other hand, in some species, male attractiveness to females (and therefore $c$; see above) may depend directly on male resource levels (or some proxy). This would result in a coupling of low $c$ values with high $R$ values. Such associations are also to be expected when $c$ and $R$ values are dependent on some other trait(s), such as a male's quality. For example, higher-quality males may be able to invest more in courtship display and/or the maintenance of a sexual ornament or position in a social hierarchy (reducing $c$ values) as well as in resources for reproduction (increasing $R$ values). All of these possibilities can be encompassed by our analysis if we express $c$ as a function of other parameters, so that $c=\left(g_{1}, g_{2}, \ldots\right)$, one of which could be $R$. Then for any $g_{i}$ if $c$ increases (decreases) with $g_{i} s^{*}$ also increases (decreases) with $g_{i}$. Thus, any factor that makes it easier for a male to obtain a mate would also cause selection to decrease his optimal ejaculate expenditure.

There is some empirical evidence consistent with the model's predictions. For example, in domestic fowl Gallus gallus domesticus (Froman et al. 2002; Cornwallis and Birkhead 2007; Pizzari et al. 2007), Arctic char Salvelinus alpinus (Rudolfsen et al. 2006), and Alpine whitefish Coregonus zugensis (Rudolfsen et al. 2008), males with privileged access to females (i.e., low $c$ ) produce ejaculates of lower fertilizing quality (controlling for number of sperm inseminated) than do subordinate males. These findings fit our predictions if sperm quality reflects investment. However, care is required to establish whether reduced ejaculate expenditure in low- $c$ males is a strategic investment by the male or arises coincidentally through sperm depletion due to higher remating rates. To test our ideas, we also need to identify species in which males do not vary greatly in the amount of effort they have to expend in order to obtain a mating. This may plausibly apply to externally fertilizing sessile organisms that broadcast gametes or to systems where males have to expend little effort to obtain matings because of female promiscuity. Under these circumstances, the model predicts that all males should invest the same amount of sperm in each mating. More empirical work is needed before strong conclusions can be drawn. Our theoretical work provides a framework for considering individual variation in ejaculate expenditure strategies.

\section{Acknowledgments}

S.J.T. is supported by an Engineering and Physical Sciences Research Council Research Studentship. We thank two anonymous reviewers for their helpful comments.

\section{APPENDIX A}

\section{Proof That Any ESS Is a Nondegenerate Maximum}

Write the fitness function (eq. [5]) in the form

$$
W(R, c, s \mid \bar{s}, \bar{n})=\frac{R}{c+s} \sum_{k=0}^{\infty} \frac{\bar{n}^{k} e^{-\bar{n}}}{k !} \frac{s}{s+k \bar{s}}=R \cdot \frac{\nu(s \mid \bar{s}, \bar{n})}{c+s},
$$

where

$$
\nu(s \mid \bar{s}, \bar{n})=e^{-\bar{n}} \sum_{k=0}^{\infty} \frac{\bar{n}^{k}}{k !} \frac{s}{s+k \vec{s}} .
$$


Clearly, for fixed $\bar{s}$ and $\bar{n}, \nu(s)$ is a sum of positive, increasing, and concave functions of $s$ and hence is positive, increasing, and concave. From equation (A1), we have

$$
W^{\prime}(s)=R \cdot \frac{(c+s) \nu^{\prime}(s)-\nu(s)}{(c+s)^{2}},
$$

which is 0 when

$$
\nu^{\prime}(s)=\frac{\nu(s)}{c+s} .
$$

Equation (A2) provides the content of the marginal-value theorem illustrated in figure 1.

At any value of $s$ for which equation (A2) holds, we have

$$
W^{\prime \prime}(s)=R \cdot \frac{(c+s)^{3} \nu^{\prime \prime}(s)-2(c+s)\left[(c+s) \nu^{\prime}(s)-\nu(s)\right]}{(c+s)^{4}}=\frac{R}{c+s} \nu^{\prime \prime}(s)<0,
$$

since $\nu(s)$ is concave. This shows that any solution of equation (A2) is a nondegenerate maximum of $W(s)$.

An ESS $s=s^{*}$ is a maximum of $W(s)$ satisfying $\bar{s}=\bar{s}^{*}$ and $\bar{n}=\bar{n}^{*}$ and hence must be a nondegenerate maximum of $W\left(R, c, s \mid \bar{s}^{*}, \bar{n}^{*}\right)$.

\section{APPENDIX B}

\section{Details of Numerical Iterative Method}

Wolfram Mathematica 6 was used to implement the following iterative numerical procedure. Here we describe the procedure for the cost-varying case; an analogous procedure was used for the resource-varying case.

First, the initial parameter value $R$ of resources each male has for mating is specified. We then suppose that the distribution of $c$ values over males is specified by a probability density function $g(c)$, typically taken to be a truncated normal distribution between fixed values $c_{\min }$ and $c_{\max }$.

To begin the procedure, an initial sperm strategy function $s_{0}(c)=1$ is chosen. The corresponding function $n_{0}(c)$ is

$$
n_{0}(c)=\frac{R}{c+s_{0}(c)}=\frac{R}{c+1} .
$$

Next, Mathematica is used to numerically integrate (using a Gauss-Kronrod quadrature method with a global adaptive strategy; Malcolm and Simpson 1975) over the interval $\left[c_{\min }, c_{\max }\right]$ to obtain $\bar{n}_{0}$,

$$
\bar{n}_{0}=\int_{c_{\min }}^{c_{\max }} \frac{R}{c+1} g(c) d c .
$$

The best reply function $s_{1}(c)$ for a male of cost $c$ in a population with $\bar{s}_{0}=1$ and $\bar{n}_{0}$, as above, is then the value that maximizes the fitness $W(R, c, s \mid \bar{s}, \bar{n})$, as defined in equation (5), given $c$ and the fixed $R$ of the population. The corresponding expected number of matings $n_{1}(c)=R /\left[c+s_{1}(c)\right]$ can then be obtained. Numerical integration is then used to calculate the means $\bar{s}_{1}$ and $\bar{n}_{1}$ : 


$$
\begin{aligned}
& \bar{s}_{1}=\int_{c_{\min }}^{c_{\max }} s_{1}(c) g(c) d c, \\
& \bar{n}_{1}=\int_{c_{\min }}^{c_{\max }} \frac{R}{s_{1}(c)+c} g(c) d c .
\end{aligned}
$$

The procedure is then iterated until solutions cease to vary by more than a specified tolerance. In our implementation, the procedure was repeated an integer number of times $i$, such that $\left|\bar{s}_{i}-\bar{s}_{i-1}\right|<10^{-4}$. The ESS was then taken to be $s_{i}(c)$, with corresponding expected number of matings $n_{i}(c)=R /\left[c+s_{i}(c)\right]$.

\section{APPENDIX C}

\section{Proof That Increasing $\bar{R}$ Increases $\bar{s}^{*}$}

Assume that the distribution of $R$ over the male population is specified by a probability density function $h(R)$. Consider the equilibrium equation

$$
\frac{c}{\left(c+s^{*}\right)} \sum_{k=0}^{\infty} \frac{\left(\bar{n}^{*}\right)^{k}}{k !} \frac{1}{s^{*}+k \bar{s}^{*}}=s^{*} \sum_{k=0}^{\infty} \frac{\left(\bar{n}^{*}\right)^{k}}{k !} \frac{1}{\left(s^{*}+k \bar{s}^{*}\right)^{2}},
$$

where $s^{*}=\bar{s}^{*}$ is independent of $R$ and

$$
\bar{n}^{*}=\int_{0}^{\infty} \frac{R}{c+s^{*}(R)} h(R) d R=\frac{\bar{R}}{c+\bar{s}^{*}} .
$$

To simplify notation, write $s^{*}=\bar{s}^{*}=\bar{s}$ and $\bar{n}^{*}=\bar{n}$. Then, equations (C1) and (C2) give two equilibrium equations:

$$
\begin{aligned}
\bar{s} \sum_{k=0}^{\infty} \frac{\bar{n}^{k}}{(k+1)(k+1) !} & =c \sum_{k=0}^{\infty} \frac{\bar{n}^{k}}{(k+1) !}\left(1-\frac{1}{k+1}\right), \\
\bar{R} & =\bar{n}(c+\bar{s}) .
\end{aligned}
$$

Note that equation (C3) defines $\bar{s}$ as a function of $\bar{n}$, and hence equation (C4) defines $\bar{R}$ as a function of $\bar{n}$. Multiplying equation (C3) through by $\bar{n}$, we can write

$$
\bar{s}=c\left[\frac{\sum_{k=0}^{\infty} \bar{n}^{k+1} /(k+1) !}{\sum_{k=0}^{\infty} \bar{n}^{k+1} /(k+1)(k+1) !}-1\right] .
$$

The expression in the numerator, $N(\bar{n})$, in equation (C5) is

$$
N(\bar{n})=e^{\bar{n}}-1,
$$

and the expression in the denominator, $D(\bar{n})$, can be written as 


$$
D(\bar{n})=\sum_{k=0}^{\infty} \bar{n}^{k+1} \frac{1}{(k+1)(k+1) !}=\int_{0}^{\bar{n}} \frac{1}{u}\left(e^{u}-1\right) d u .
$$

Thus, from equations (C5)-(C7) we obtain $\bar{s}$ as a function of $\bar{n}$ :

$$
\bar{s}(\bar{n})=c\left[\frac{N(\bar{n})}{D(\bar{n})}-1\right]=c\left[\frac{e^{\bar{n}}-1}{\int_{0}^{\bar{n}}(1 / u)\left(e^{u}-1\right) d u}-1\right] .
$$

We show below that $\bar{s}(\bar{n})$ is monotonically increasing in $\bar{n}$. Note that $N(\bar{n}) / D(\bar{n}) \rightarrow 1$ as $\bar{n} \rightarrow 0$ (by L'Hôpital's rule), and hence $\bar{s}(0)=0$.

It follows from equation (C4) that, if $\bar{s}(\bar{n})$ is a monotonically increasing function of $\bar{n}$, then so is $\bar{R}(\bar{n})$. Thus, $d \bar{s} / d \bar{R}=(d \bar{s} / d \bar{n}) /(d \bar{R} / d \bar{n})$ is positive. This gives the required result that $\bar{s}$ increases as $\bar{R}$ increases.

It remains to show that $\bar{s}(\bar{n})$ is a monotonically increasing function of $\bar{n}$. From equation (C8), we are required to show that $N(\bar{n}) / D(\bar{n})$ is increasing. Since $N(\bar{n})$ and $D(\bar{n})$ are both nonnegative, this is the case if

$$
\frac{N^{\prime}(\bar{n})}{N(\bar{n})} \geq \frac{D^{\prime}(\bar{n})}{D(\bar{n})}
$$

with equality only if $\bar{n}=0$. That is, using equations (C6) and (C7),

$$
D(\bar{n}) \geq \frac{1}{\bar{n}}\left(e^{\bar{n}}+e^{-\bar{n}}-1\right),
$$

with equality only if $\bar{n}=0$.

To verify equation (C9), consider the function $F(\bar{n})=D(\bar{n})-(1 / \bar{n})\left(e^{\bar{n}}+e^{-\bar{n}}-2\right)$. Then, $F(0)=0$, and

$$
\begin{aligned}
F^{\prime}(\bar{n}) & =\frac{1}{\bar{n}}\left(e^{\bar{n}}-1\right)-\frac{1}{\bar{n}}\left(e^{\bar{n}}-e^{-\bar{n}}\right)+\frac{1}{\bar{n}^{2}}\left(e^{\bar{n}}+e^{-\bar{n}}-2\right) \\
& =\frac{1}{\bar{n}^{2}}\left(1-e^{-\bar{n}}\right)\left[e^{\bar{n}}-(1+\bar{n})\right] \\
& \geq 0,
\end{aligned}
$$

with equality only when $\bar{n}=0$. Thus, $F(\bar{n})$ is monotonically increasing for $\bar{n}>0$, which proves equation (C9) and hence gives the required result.

\section{Literature Cited}

Alcock, J. 1994. Postinsemination associations between males and females in insects: the mate-guarding hypothesis. Annual Review of Entomology 39:1-21.

Alonzo, S. H., and R. R. Warner. 1999. A trade-off generated by sexual conflict: Mediterranean wrasse males refuse present mates to increase future success. Behavioral Ecology 10:105-111.

Andersson, M. 1994. Sexual selection. Princeton University Press, Princeton, NJ.

Andersson, M., and Y. Iwasa. 1996. Sexual selection. Trends in Ecology \& Evolution 11:A53-A58.

Ball, M. A., and G. A. Parker. 2000. Sperm competition games: a comparison of loaded raffle models and their biological implications. Journal of Theoretical Biology 206:487-506.
Charnov, E. L. 1976. Optimal foraging, the marginal value theorem. Theoretical Population Biology 9:129-136.

Clutton-Brock, T. H., and G. A. Parker. 1995. Punishment in animal societies. Nature 373:209-216.

Cook, P. A., and N. Wedell. 1999. Non-fertile sperm delay female remating. Nature 397:486.

Cornwallis, C. K., and T. R. Birkhead. 2007. Changes in sperm quality and numbers in response to experimental manipulation of male social status and female attractiveness. American Naturalist 170: 758-770.

Froman, D. P., T. Pizzari, A. J. Feltmann, H. Castillo-Juarez, and T. R. Birkhead. 2002. Sperm mobility: mechanisms of fertilizing efficiency, genetic variation and phenotypic relationship with male status in the domestic fowl, Gallus gallus domesticus. Proceedings of the Royal Society B: Biological Sciences 269:607-612.

Gage, M. J. G., and P. A. Cook. 1994. Sperm size or numbers? effects of nutritional stress upon eupyrene and apyrene sperm production 


\section{E82 The American Naturalist}

strategies in the moth Plodia interpunctella (Lepidoptera, Pyralidae). Functional Ecology 8:594-599.

Gage, M. J. G., and E. H. Morrow. 2003. Experimental evidence for the evolution of numerous, tiny sperm via sperm competition. Current Biology 13:754-757.

Gavrilets, S., and T. I. Hayashi. 2006. The dynamics of two- and three-way sexual conflicts over mating. Philosophical Transactions of the Royal Society B: Biological Sciences 361:345-354.

Graves, B. M., and D. Duvall. 1995. Effects of sexually transmitted diseases on heritable variation in sexually selected systems. Animal Behaviour 50:1129-1131.

Jennions, M. D., and M. Petrie. 2000. Why do females mate multiply? a review of the genetic benefits. Biological Reviews 75:21-64.

Johnstone, R. A., and L. Keller. 2000. How males can gain by harming their mates: sexual conflict, seminal toxins, and the cost of mating. American Naturalist 156:368-377.

Lessells, C. M. 2005. Why are males bad for females? models for the evolution of damaging male mating behavior. American Naturalist 165(suppl.):S46-S63.

Løvlie, H., C. K. Cornwallis, and T. Pizzari. 2005. Male mounting alone reduces female promiscuity in the fowl. Current Biology 15: 1222-1227.

Malcolm, M. A., and R. B. Simpson. 1975. Local versus global strategies for adaptive quadrature. ACM Transactions on Mathematical Software 1:129-146.

Martin, P. A., T. J. Reimers, J. R. Lodge, and P. J. Dziuk. 1974. The effect of ratios and numbers of spermatozoa mixed from two males on proportions of offspring. Journal of Reproduction and Fertility 39:251-258.

Maynard Smith, J. 1974. Theory of games and evolution of animal conflicts. Journal of Theoretical Biology 47:209-221.

- 1982. Evolution and the theory of games. Cambridge University Press, Cambridge.

Mesterton-Gibbons, M. 1999a. On sperm competition games: incomplete fertilization risk and the equity paradox. Proceedings of the Royal Society B: Biological Sciences 266:269-274.

. 1999b. On sperm competition games: raffles and roles revisited. Journal of Mathematical Biology 39:91-108.

Moore, A. J., and T. Pizzari. 2005. Quantitative genetic models of sexual conflict based on interacting phenotypes. American Naturalist 165(suppl.):S88-S97.

Moreira, P. L., V. L. Nunes, J. Martín, and O. S. Paulo. 2007. Copulatory plugs do not assure high first male fertilisation success: sperm displacement in a lizard. Behavioral Ecology and Sociobiology 62:281-288.

Parker, G. A. 1970. Sperm competition and its evolutionary consequences in the insects. Biological Reviews of the Cambridge Philosophical Society 45:525-567.

. 1982. Why are there so many tiny sperm? sperm competition and the maintenance of two sexes. Journal of Theoretical Biology 96:281-294.

. 1990a. Sperm competition games: raffles and roles. Proceedings of the Royal Society B: Biological Sciences 242:120-126. 1990b. Sperm competition games: sneaks and extra-pair copulations. Proceedings of the Royal Society B: Biological Sciences 242:127-133.

. 1998. Sperm competition and the evolution of ejaculates: towards a theory base. Pages 3-54 in T. R. Birkhead and A. P. Møller, eds. Sperm competition and sexual selection. Academic Press, San Diego, CA.

Parker, G. A., and M. A. Ball. 2005. Sperm competition, mating rate and the evolution of testis and ejaculate sizes: a population model. Biology Letters 1:235-238.

Parker, G. A., M. A. Ball, P. Stockley, and M. J. G. Gage. 1996. Sperm competition games: individual assessment of sperm competition intensity by group spawners. Proceedings of the Royal Society B: Biological Sciences 263:1291-1297.

Pianka, E. R., and W. S. Parker. 1975. Age-specific reproductive tactics. American Naturalist 109:453-464.

Pizzari, T., and K. R. Foster. 2008. Sperm sociality: cooperation, altruism, and spite. PLoS Biology 6:925-931.

Pizzari, T., and G. A. Parker. 2009. Sperm competition and sperm phenotype. Pages 207-245 in T. R. Birkhead, D. J. Hosken, and S. Pitnick, eds. Sperm biology: an evolutionary perspective. Academic Press, London.

Pizzari, T., and R. R. Snook. 2004. Sexual conflict and sexual selection: measuring antagonistic coevolution. Evolution 58:1389-1393.

Pizzari, T., C. K. Cornwallis, and D. P. Froman. 2007. Social competitiveness associated with rapid fluctuations in sperm quality in male fowl. Proceedings of the Royal Society B: Biological Sciences 274:853-860.

Ram, K. R., and M. F. Wolfner. 2007. Sustained post-mating response in Drosophila melanogaster requires multiple seminal fluid proteins. PLoS Genetics 3:2428-2438.

Rudolfsen, G., L. Figenschou, I. Folstad, H. Tveiten, and M. Figenschou. 2006. Rapid adjustments of sperm characteristics in relation to social status. Proceedings of the Royal Society B: Biological Sciences 273:325-332.

Rudolfsen, G., R. Muller, D. Urbach, and C. Wedekind. 2008. Predicting the mating system from phenotypic correlations between life-history and sperm quality traits in the Alpine whitefish Coregonus zugensis. Behavioral Ecology and Sociobiology 62:561-567.

Shuster, S. M., and M. J. Wade. 2003. Mating systems and strategies. Princeton University Press, Princeton, NJ.

Simmons, L. W. 2001. Sperm competition and its evolutionary consequences in the insects. Princeton University Press, Princeton, NJ.

Warner, R. R., D. Y. Shapiro, A. Marcanato, and C. W. Petersen. 1995. Sexual conflict: males with highest mating success convey the lowest fertilization benefits to females. Proceedings of the Royal Society B: Biological Sciences 262:135-139.

Wedell, N., M. J. G. Gage, and G. A. Parker. 2002. Sperm competition, male prudence and sperm-limited females. Trends in Ecology \& Evolution 17:313-320.

Williams, P. D., T. Day, and E. Cameron. 2005. The evolution of sperm-allocation strategies and the degree of sperm competition. Evolution 59:492-499.

Associate Editor: Peter D. Taylor Editor: Michael C. Whitlock 